\title{
Electric-field-induced two- dimensional hole gas in undoped GaSb quantum wells
}

\section{Journal Article}

\section{Author(s):}

Shibata, Kenji; Karalic, Matija; Mittag, Christopher; Tschirky, Thomas; Reichl, Christian; Ito, H.; Hashimoto, Koichi; Tomimatsu, T.; Hirayama, Yoshiro; Wegscheider, Werner (D); Ihn, Thomas Markus (D); Ensslin, Klaus (D)

Publication date:

2019-06-10

\section{Permanent link:}

https://doi.org/10.3929/ethz-b-000348882

\section{Rights / license:}

In Copyright - Non-Commercial Use Permitted

\section{Originally published in:}

Applied Physics Letters 114(23), https://doi.org/10.1063/1.5093133 


\section{Electric-field-induced two-dimensional hole gas in undoped GaSb quantum wells}

Cite as: Appl. Phys. Lett. 114, 232102 (2019); https://doi.org/10.1063/1.5093133

Submitted: 18 February 2019 . Accepted: 29 May 2019 . Published Online: 11 June 2019

K. Shibata, M. Karalic, C. Mittag, (D) T. Tschirky, C. Reichl, H. Ito, K. Hashimoto, T. Tomimatsu, (D) Y. Hirayama, W. Wegscheider, T. Ihn, and K. Ensslin
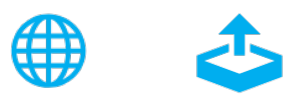

\section{ARTICLES YOU MAY BE INTERESTED IN}

Quantum transport in high-quality shallow InSb quantum wells

Applied Physics Letters 115, 012101 (2019); https://doi.org/10.1063/1.5098294

Exciton aggregation induced photoluminescence enhancement of monolayer WS 2

Applied Physics Letters 114, 232101 (2019); https://doi.org/10.1063/1.5096206

Effects of gas cluster ion beam sputtering on the molecular orientation of organic semiconductor films: Ultraviolet photoelectron spectroscopy study of [6]phenacene

Applied Physics Letters 114, 231603 (2019); https://doi.org/10.1063/1.5094952

\section{Challenge us.}

What are your needs for periodic signal detection?

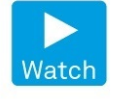

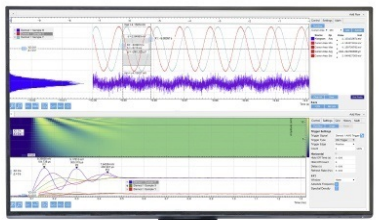

-
Zurich

Instruments 


\title{
Electric-field-induced two-dimensional hole gas in undoped GaSb quantum wells
}

\author{
Cite as: Appl. Phys. Lett. 114, 232102 (2019); doi: 10.1063/1.5093133 \\ Submitted: 18 February 2019 - Accepted: 29 May 2019 . \\ Published Online: 11 June 2019
}

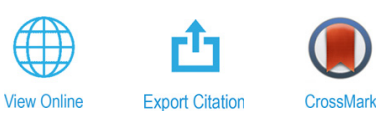

K. Shibata, ${ }^{1,2, a)}$ M. Karalic, ${ }^{7}$ C. Mittag, ${ }^{7}$ T. Tschirky, ${ }^{7}$ (D) C. Reichl, ${ }^{1}$ H. Ito, ${ }^{2}$ K. Hashimoto, ${ }^{3,4}$ T. Tomimatsu, $^{3}$ Y. Hirayama, ${ }^{3,4,5}$ (D) W. Wegscheider, T. Ihn, and K. Ensslin ${ }^{\top}$

\author{
AFFILIATIONS \\ ${ }^{7}$ Solid State Physics Laboratory, ETH Zurich, 8093 Zurich, Switzerland \\ ${ }^{2}$ Tohoku Institute of Technology, Sendai 982-8577, Japan \\ ${ }^{3}$ Craduate School of Science, Tohoku University, Sendai 980-8578, Japan \\ ${ }^{4}$ Center for Spintronics Research Network, Tohoku University, Sendai 980-8577, Japan \\ ${ }^{5}$ Center for Science and Innovation in Spintronics (Core Research Cluster), Tohoku University, Sendai 980-8577, Japan

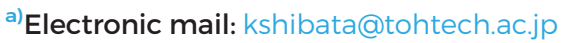

\begin{abstract}
We have measured hole transport in electrically induced two-dimensional hole gases in undoped GaSb/AlSb quantum wells. In order to access the electrically induced two-dimensional hole gas in GaSb quantum wells, recessed ohmic contacts were formed and the lowtemperature magnetoresistance was measured for a gate-defined Hall bar geometry. The mobility of the sample increases with increasing hole density and reaches $20000 \mathrm{~cm}^{2} / \mathrm{V}$ s at a hole density of $5.3 \times 10^{11} \mathrm{~cm}^{-2}$ for an 8 -nm-thick GaSb quantum well. The longitudinal and Hall resistivities show Shubnikov-de Haas oscillations and integer quantum Hall plateaus, respectively. These results establish a platform for realizing spin-based electronics using the strong spin-orbit interaction of this material and are also useful for understanding the transport properties of the two-dimensional topological insulator realized in InAs/GaSb double quantum well structures.
\end{abstract}

Published under license by AIP Publishing. https://doi.org/10.1063/1.5093133

GaSb is a III-V compound semiconductor with a bandgap of $\sim 0.7 \mathrm{eV}$. It has high hole mobility at room temperature (RT) and is considered as an alternative channel material to $\mathrm{Si}^{1-11} \mathrm{GaSb}$ is also known as one of the base materials of InAs/GaSb double quantum wells (QWs), which have been intensively studied for their application in mid-infrared photodetectors ${ }^{12}$ and have more recently received increasing attention in the context of topological insulators. ${ }^{13-19}$ In an InAs/GaSb double QW, owing to the band alignment between InAs and $\mathrm{GaSb}$, it has been relatively easy to induce holes in the GaSb layer by applying negative gate voltages. So far, two-dimensional (2D) hole transport in the GaSb layer in InAs/GaSb double QW structures at cryogenic temperatures has been investigated. ${ }^{20-22}$ Transport through doped GaSb QWs has recently been reported by some of the authors. ${ }^{23}$ However, there have been no reports on 2D hole transport in undoped GaSb QWs at cryogenic temperatures. A thorough understanding will be beneficial for spintronics applications and also for studying InAs/GaSb double QW systems. In this study, we have investigated transport of electrically induced holes in GaSb QWs at cryogenic temperatures.
In GaSb/AlSb heterostructures, modulation doping has been used to enhance RT mobility ${ }^{5-7}$ and there are few reports on the resulting low-temperature transport properties. ${ }^{23,24}$ In this work, therefore, we developed a method of electrically inducing a $2 \mathrm{D}$ hole gas (2DHG) in undoped GaSb/AlSb QWs. We formed recessed ohmic contacts $^{25-28}$ to contact the electrically induced hole gas.

Figure 1(a) shows a schematic of the wafer structure used in this study. GaSb/AlSb heterostructures were grown in the [100] direction on undoped GaSb substrates by molecular beam epitaxy. We measured samples with 8- and 25-nm-thick GaSb channel layers sandwiched between AlSb barrier layers. The thickness of the upper AlSb barrier layer was $20 \mathrm{~nm}$, while that of the lower barrier was $100 \mathrm{~nm}$. The topmost GaSb capping layer should be sufficiently thin to prevent additional and undesired hole accumulation which would screen the gate electric field. In this work, we measured the hole transport for samples with $\sim 1$ - and 3-nm-thick GaSb capping layers. Figure 1(b) shows a schematic cross-sectional image of a fabricated device. To form recessed ohmic contacts, we first wet-etched the wafer using phosphoric acid and citric acid-based III-V etchants. ${ }^{29}$ The etching 
(a)

\begin{tabular}{|c|}
\hline GaSb (capping layer) \\
\hline AlSb $20 \mathrm{~nm}$ \\
\hline GaSb (8 $\mathrm{nm}$ or $25 \mathrm{~nm}$ ) \\
\hline AlSb $100 \mathrm{~nm}$ \\
\hline GaSb/AlSb Superlattice \\
\hline GaSb buffer layer \\
\hline Nondoped GaSb substrate \\
\hline
\end{tabular}

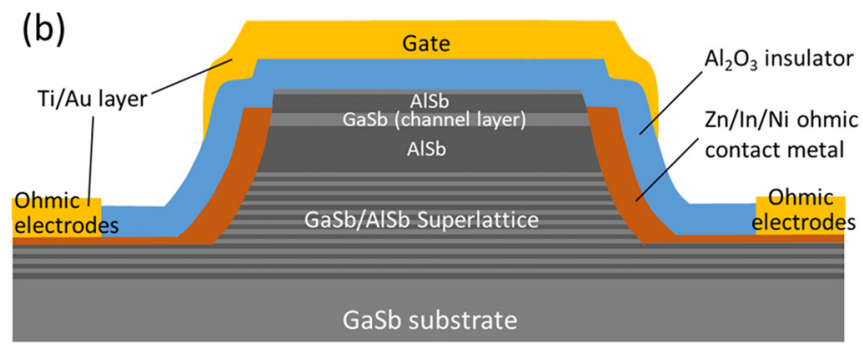

FIG. 1. (a) Schematic wafer structure. (b) Schematic cross-sectional image of the device structure along the line $A B$ in (c). Recessed ohmic contacts are formed to electrically access the undoped GaSb QW channel layer. (c) Optical microscopy image of the sample (top view)

depth was about $500 \mathrm{~nm}$. Then, the ohmic contact metal, Ti $(10 \mathrm{~nm}) /$ $\mathrm{Zn}(20 \mathrm{~nm}) / \mathrm{In}(100 \mathrm{~nm}) / \mathrm{Ni}(50 \mathrm{~nm})$, for the hole transport was deposited at a large tilt angle $\left(\sim 80^{\circ}\right.$ tilt angle measured from the sample normal) to deposit the metal on the side wall of the mesa structure. Roughness at the edge of the ohmic contact metal leads to gate leakage; thus, particular attention was paid to making the edges of the ohmic contact metal smooth (see Figs. S1-S3 in the supplementary material). After the removal of the photoresist, the samples were annealed in a forming gas at $400{ }^{\circ} \mathrm{C}$ to alloy the ohmic contact. Next, a gate insulating $\mathrm{Al}_{2} \mathrm{O}_{3}$ layer ( $80 \mathrm{~nm}$ thickness) was deposited by atomic layer deposition, and finally, $\mathrm{Ti} / \mathrm{Au}$ layers were added by evaporation serving as the gate and ohmic electrodes. The ohmic electrodes had an electrical connection to the channel GaSb layer through the $\mathrm{Ti} / \mathrm{Zn} / \mathrm{In} / \mathrm{Ni}$ ohmic contact metal, as shown in Fig. 1(b). Figure 1(c) shows an optical microscopy image of a fabricated sample (top view). The samples are Hall bars (width $100 \mu \mathrm{m}$ and length $450 \mu \mathrm{m}$ ) and the transport channel is defined by the gate electrodes. The magnetotransport properties were measured in the dark in both a variable-temperature insert cryostat and a dilution refrigerator using a standard low-frequency $(13 \mathrm{~Hz})$ lock-in technique at a constant excitation voltage of $1 \mathrm{mV}$. The magnetic field, $B$, was applied perpendicular to the sample surface.

Figure 2 shows the transfer characteristics of the fabricated samples measured at $4.0 \mathrm{~K}$. The source-drain current, $I_{\mathrm{sd}}$, increases with decreasing gate voltage $V_{\mathrm{G}}$, suggesting that holes are induced in the GaSb QW and ohmic contacts to the channel layer are formed. We also confirmed that $I_{\text {sd }}$ increases linearly with the increasing sourcedrain voltage, $V_{\text {sd. }}$. The resistance of the samples before the increase in (a) $3 \mathrm{~nm}$ GaSb capping

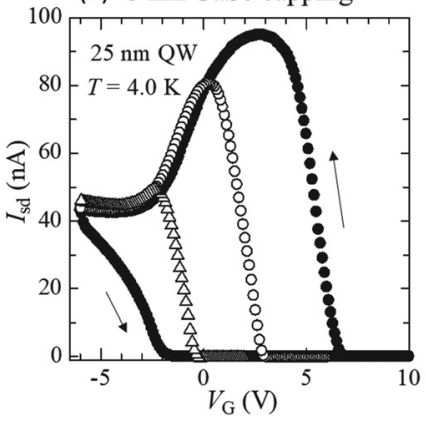

(b) $\sim 1 \mathrm{~nm}$ GaSb capping

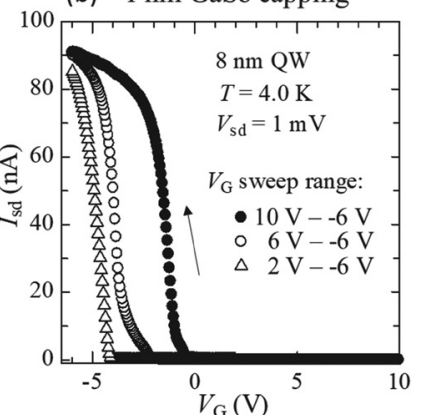

FIG. 2. Transfer characteristics of samples with capping layer thicknesses of (a) $3 \mathrm{~nm}(25 \mathrm{~nm} \mathrm{QW})$ and $(\mathrm{b}) \sim 1 \mathrm{~nm}(8 \mathrm{~nm} \mathrm{QW})$ plotted for various sweep ranges of $V_{G}$. The sweep ranges of $V_{G}$ for both figures are indicated in (b). The data are shown only for the decreasing branch of the $V_{G}$ sweep, except for the closed circles in (a). A peak structure similar to (a) is observed also for samples with a capping layer thickness of $3 \mathrm{~nm}(8 \mathrm{~nm} \mathrm{QW})$, as shown in Fig. $S 4(b)$ in the supplementary material.

$I_{\text {sd }}$ is typically $\sim 10 \mathrm{M} \Omega$, meaning that the samples are insulating in the absence of charge carriers in the QW. The transfer characteristics always show hysteresis between currents measured with increasing and decreasing gate voltage [see closed circles in Figs. 2(a) and S4 in the supplementary material], suggesting trapping of charges in the gate dielectric layers. Figure 2(a) shows $I_{\text {sd }}$ as a function of $V_{\mathrm{G}}$ plotted for various gate sweep ranges for samples with the 3-nm-thick capping layer. The data are plotted for the decreasing branch of the gate voltage sweep [the increasing branch is shown only for the closed circles in Fig. 2(a)]. For the samples with the 3-nm-thick capping layer and $25 \mathrm{~nm}$ QW width, the transfer curves show a clear peak structure and strongly depend on the gate sweep range, as shown in Fig. 2(a). A similar peak structure is also observed for 8-nm-thick QW, as shown in Fig. S4(b) in the supplementary material. On the other hand, if the capping layer is wet-etched down to $\sim 1 \mathrm{~nm}$ thickness, although the transfer curves slightly shift with the gate sweep range, only a monotonic increase in $I_{\mathrm{sd}}$ was observed with decreasing $V_{\mathrm{G}}$, as shown in Figs. 2(b) and S5 in the supplementary material. For the samples with the 3-nm-thick capping layer, the hole density and mobility also show a $V_{\mathrm{G}}$ dependence similar to $I_{\mathrm{sd}}$ (see Figs. S6 and S7 in the supplementary material), indicating that the accessible hole density depends on the gate voltage sweep range. This behavior might be related to the charge trapping in the gate dielectric layer in our sample. In particular, the 3-nm-thick GaSb capping layer could act as a holetrapping layer. Since the holes might have a large effective mass, a capping layer thickness of $3 \mathrm{~nm}$ is sufficient to form a hole quantum state above the Fermi energy. The holes induced in the capping layers screen the gate electric field and prevent the increase in the hole density or even force a decrease in the hole density in the channel layer. Since the capping layer is not directly connected to the ohmic contacts in our sample [see Figs. 1(b), S2, and S3 in the supplementary material], the holes transfer to the capping layer by tunneling from the gate or channel. When the $V_{\mathrm{G}}$-sweep is started at a large positive value, no holes exist in the capping layer and the hole density in the channel increases to its maximum value. However, when the $V_{\mathrm{G}}$-sweep is started at negative values, a large number of holes are already trapped in the capping layer from the beginning, preventing an increase in the 
hole density in the channel. To investigate hole transport for a wide range of hole densities, we always started $V_{\mathrm{G}}$ sweeps at large positive values for the samples with the 3-nm-thick capping layer. For simulations of the band profile and the hole density in both the QW and the capping layer, see Secs. 5 and 6 in the supplementary material.

Figure 3(a) shows the hole density as a function of the gate voltage measured from the onset of $I_{\text {sd }}$. For both samples, the hole density increases with sweeping $V_{\mathrm{G}}$ in the negative direction. The slope of the increase in the hole density agrees with the slope calculated using the parallel plate capacitor model between gate electrodes and the channel (dashed red line), indicating that holes are electrically induced in the GaSb channel layer (see Fig. S13 in the supplementary material). The hole density tends to saturate at a high negative $V_{\mathrm{G}}$, which might be explained by hole trapping in the gate insulator layers and/or hole accumulation at the GaSb capping layer. Figure 3(b) shows mobility vs hole density for 8- and 25-nm-thick GaSb QWs. For the 8-nm-thick QW, the mobility reaches $20000 \mathrm{~cm}^{2} / \mathrm{Vs}$ at a hole density of $5.3 \times 10^{11} \mathrm{~cm}^{-2}$. On the other hand, for the 25-nm-thick QW, we can access a higher density. The mobility in the $25-\mathrm{nm}$-thick QW is lower than that in the 8-nm-thick QW (see also Sec. 8 in the supplementary material).

Figure 4(a) shows the magnetic field dependence of the magnetoresistance, $\rho_{\mathrm{xx}}$ (open circles), and Hall resistance, $\rho_{\mathrm{xy}}$ (closed circles), at a hole density of $5.3 \times 10^{11} \mathrm{~cm}^{-2}$ for the 8-nm-thick QW $(\sim 1-\mathrm{nm}$ thick capping layer) measured at $1.5 \mathrm{~K}$. Clear Shubnikov-de Haas (SdH) oscillations are observed in $\rho_{\mathrm{xx}}$ at a low magnetic field, which eventually evolve to quantum Hall behavior with plateaus in $\rho_{\mathrm{xy}}$ and vanishing $\rho_{\mathrm{xx}}$ at a high magnetic field. The red numbers indicate the corresponding filling factors, $\nu$, of the quantum Hall states. The spinsplitting of Landau levels is clearly resolved, which gives rise to the appearance of even and odd filling factors. Figure 4(b) shows the gate voltage dependence of $\rho_{\mathrm{xx}}$ and $\rho_{\mathrm{xy}}$ measured at $B=8 \mathrm{~T} ; \rho_{\mathrm{xx}}$ clearly vanishes at integer filling factors (for the magnetotransport properties in the $25 \mathrm{~nm}$ QW, see Fig. S14 in the supplementary material). We emphasize that there was no intentional doping of the sample and the $2 \mathrm{D}$ holes were purely induced by the applied $V_{\mathrm{G}}$, which reduces scattering by ionized impurities, and a higher mobility was achieved than
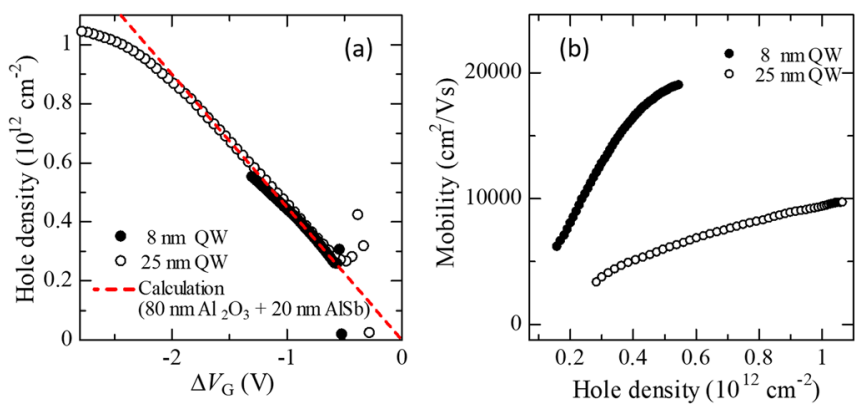

FIG. 3. (a) Hole density as a function of the gate voltage measured from the onset of $I_{s d}, \Delta V_{G}$, extracted from the classical Hall measurements at $1.5 \mathrm{~K}$. The dashed red line shows the slope calculated using a parallel plate capacitor model between gate electrodes and the channel. (b) Mobility as a function of the hole density for samples with 8- and 25-nm-thick QWs. For the $25 \mathrm{~nm}$ QW (3-nm-thick capping layer), the data in the range of $4 \mathrm{~V} \leq V_{\mathrm{G}} \leq 6.5 \mathrm{~V}$ of the closed circles in Fig. 2(a) are plotted. For the $8 \mathrm{~nm} \mathrm{QW}$ ( $\sim 1$-nm-thick capping layer), the data in the range of $-6 \mathrm{~V}<\mathrm{V}_{\mathrm{G}}<-4.5 \mathrm{~V}$ of the open triangles in Fig. 2(b) are plotted.

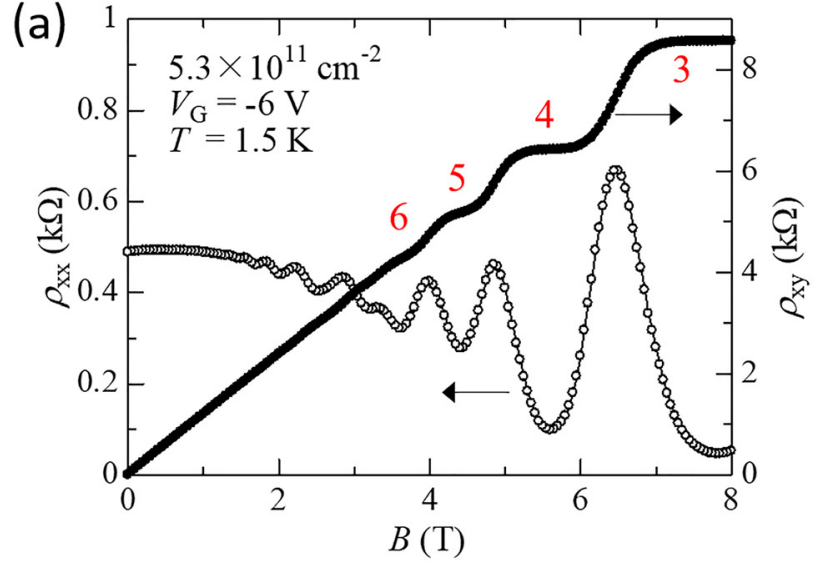

(b)

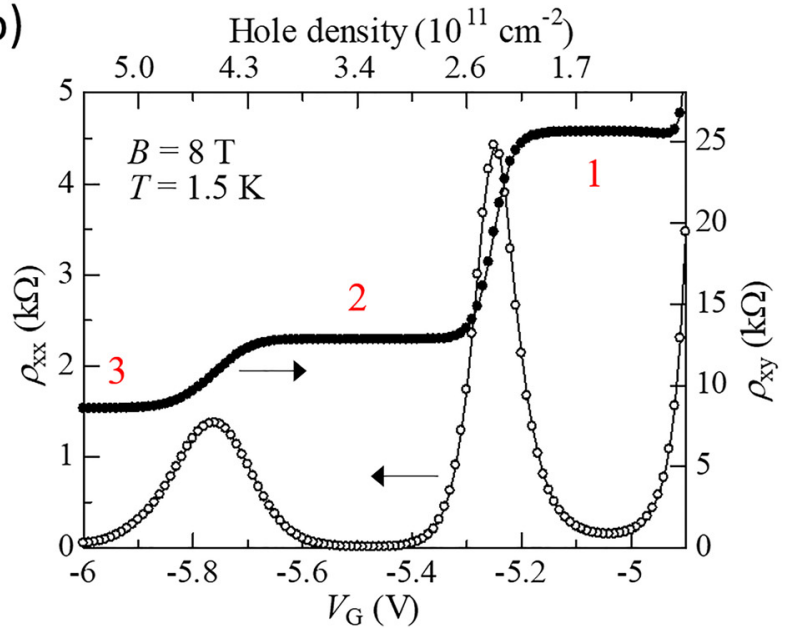

FIG. 4. (a) $\rho_{\mathrm{xx}}$ (open circles) and $\rho_{\mathrm{xy}}$ (closed circles) as a function of the magnetic field measured for a hole density of $5.3 \times 10^{11} \mathrm{~cm}^{-2}$. (b) $\rho_{\mathrm{xx}}$ and $\rho_{\mathrm{xy}}$ as a function of the gate voltage measured at $B=8 \mathrm{~T}$. The red numbers indicate the corresponding filling factors, $\nu$, of the quantum Hall states.

that reported previously for modulation-doped samples at cryogenic temperatures. ${ }^{2,24}$ This results in the clear observation of the quantum Hall effect in the GaSb QWs in this study. In low-temperature 2D hole transport, p-type GaAs QWs and SiGe heterostructures have been extensively studied. ${ }^{30,31}$ Although the GaSb QWs presented here have lower hole mobility, the hole mobility of GaSb has potential to be enhanced through strain engineering ${ }^{32}$ using the high receptivity of GaSb to strain. ${ }^{33}$ Moreover, GaSb exhibits a stronger spin-orbit interaction than the GaAs and SiGe systems. ${ }^{34}$ In this work, we provide a platform for realizing a 2DHG in GaSb/AlSb heterostructures, which will be useful for realizing spin-based electronics using the stronger spin-orbit interaction of this material and also for understanding the transport properties of the 2D topological insulator realized in InAs/ GaSb double QW structures.

In summary, we have investigated the transport properties of electrically induced 2DHGs in undoped GaSb QWs. Recessed ohmic contacts were formed, and the low-temperature magnetotransport was measured. The longitudinal and Hall resistivities show $\mathrm{SdH}$ oscillations and integer quantum Hall plateaus. These results indicate the 
formation and characterization of electrically induced 2DHGs in GaSb QWs.

See the supplementary material for the additional characterization analysis of the GaSb QWs.

The authors acknowledge the support of the ETH FIRST Laboratory and the financial support of the Swiss Science Foundation (Schweizerischer Nationalfonds, NCCR QSIT). K.S. thanks E. Cheah for his support on the band profile simulation. This work was partly supported by Grants-in-Aid from JSPS (Nos. 15KK0215 and 17H02732).

\section{REFERENCES}

${ }^{1}$ P. S. Dutta, H. L. Bhat, and V. Kumar, J. Appl. Phys. 81, 5821 (1997).

${ }^{2}$ B. R. Bennett, R. Magno, J. B. Boos, W. Kruppa, and M. G. Ancona, Solid-State Electron. 49, 1875 (2005).

${ }^{3}$ C. Merckling, X. Sun, A. Alian, G. Brammertz, V. V. Afanas'ev, T. Y. Hoffmann, M. Heyns, M. Caymax, and J. Dekoster, J. Appl, Phys. 109, 073719 (2011).

${ }^{4}$ A. Nainani, T. Irisawa, Z. Yuan, B. R. Bennett, J. B. Boos, Y. Nishi, and K. C. Saraswat, IEEE Trans. Electron Devices 58, 3407 (2011).

${ }^{5}$ B. R. Bennett, M. G. Ancona, J. B. Boos, C. B. Canedy, and S. A. Khan, J. Cryst. Growth 311, 47 (2008).

${ }^{6}$ V. Tokranov, P. Nagaiah, M. Yakimov, R. J. Matyi, and S. Oktyabrsky, J. Cryst. Growth 323, 35 (2011).

${ }^{7}$ B. R. Bennett, T. F. Chick, M. G. Ancona, and J. Brad Boos, Solid-State Electron. 79, 274 (2013).

${ }^{8}$ M. Jeppsson, K. A. Dick, H. A. Nilsson, N. Sköld, J. B. Wagner, P. Caroff, and L.-E. Wernersson, J. Cryst. Growth 310, 5119 (2008).

${ }^{9}$ Z.-X. Yang, N. Han, M. Fang, H. Lin, H.-Y. Cheung, S. Yip, E.-J. Wang, T, Hung, C.-Y. Wong, and J. C. Ho, Nat. Commun. 5, 5249 (2014).

${ }^{10}$ Z.-X. Yang, S. Yip, D. Li, N. Han, G. Dong, X. Liang, L. Shu, T. F. Hung, X. Mo, and J. C. Ho, ACS Nano 9, 9268 (2015).

${ }^{11}$ M. Borg, H. Schmid, J. Gooth, M. D. Rossell, D. Cutaia, M. Knoedler, N. Bologna, S. Wirths, K. E. Moselund, and H. Riel, ACS Nano 11, 2554 (2017).

${ }^{12}$ M. Razeghi, S. Abdollahi Pour, E. K. Huang, G. Chen, A. Haddadi, and B. M. Nguyen, Opto-Electron. Rev. 19, 261-269 (2011).
${ }^{13}$ C. L. Kane and E. J. Mele, Phys. Rev. Lett. 95, 146802 (2005).

${ }^{14}$ C. L. Kane and E. J. Mele, Phys. Rev. Lett. 95, 226801 (2005).

${ }^{15}$ B. A. Bernevig and S.-C. Zhang, Phys. Rev. Lett. 96, 106802 (2006).

${ }^{16}$ C. Liu, T. L. Hughes, X.-L. Qi, K. Wang, and S.-C. Zhang, Phys. Rev. Lett. 100, 236601 (2008).

${ }^{17}$ K. Suzuki, Y. Harada, K. Onomitsu, and K. Muraki, Phys. Rev. B 87, 235311 (2013).

${ }^{18}$ L. Du, I. Knez, G. Sullivan, and R.-R. Du, Phys. Rev. Lett. 114, 096802 (2015).

${ }^{19}$ S. Müller, A. N. Pal, M. Karalic, T. Tschirky, C. Charpentier, W. Wegscheider, K. Ensslin, and T. Ihn, Phys. Rev. B 92, 081303 (2015).

${ }^{20}$ X. Mu, G. Sullivan, and R. R. Du, Appl. Phys. Lett. 108, 012101 (2016).

${ }^{21}$ C. S. Knox, C. Morrison, F. Herling, D. A. Ritchie, O. Newell, M. Myronov, E. H. Linfield, and C. H. Marrows, Semicond. Sci. Technol. 32, 104002 (2017).

${ }^{22}$ F. Nichele, A. N. Pal, P. Pietsch, T. Ihn, K. Ensslin, C. Charpentier, and W. Wegscheider, Phys. Rev. Lett. 112, 036802 (2014).

${ }^{23}$ M. Karalic, C. Mittag, M. Hug, K. Shibata, T. Tschirky, W. Wegscheider, R. Winkler, K. Ensslin, and T. Ihn, Phys. Rev. B 99, 115435 (2019).

${ }^{24}$ L. Hanks, M. Hayne, A. R. J. Marshall, and L. Ponomarenko, J. Phys.: Conf. Ser. 964, 012006 (2018).

${ }^{25}$ R. L. Willett, L. N. Pfeiffer, and K. W. West, Appl. Phys. Lett. 89, 242107 (2006).

${ }^{26}$ T. M. Lu, D. R. Luhman, K. Lai, D. C. Tsui, L. N. Pfeiffer, and K. W. West, Appl. Phys. Lett. 90, 112113 (2007).

${ }^{27}$ S. Sarkozy, K. Das Gupta, F. Sfigakis, I. Farrer, H. Beere, R. Harrell, D. Ritchie, and G. Jones, ECS Trans. 11, 75 (2007).

${ }^{28}$ S. Sarkozy, K. Das Gupta, C. Siegert, A. Ghosh, M. Pepper, I. Farrer, H. E. Beere, D. A. Ritchie, and G. A. C. Jones, Appl. Phys. Lett. 94, 172105 (2009).

${ }^{29}$ A. N. Pal, S. Müller, T. Ihn, K. Ensslin, T. Tschirky, C. Charpentier, and W. Wegscheider, AIP Adv. 5, 077106 (2015).

${ }^{30}$ F. Nichele, A. N. Pal, R. Winkler, C. Gerl, W. Wegscheider, T. Ihn, and K. Ensslin, Phys. Rev. B 89, 081306(R) (2014); and references therein.

${ }^{31}$ A. Dobbie, M. Myronov, R. J. H. Morris, A. H. A. Hassan, M. J. Prest, V. A. Shah, E. H. C. Parker, T. E. Whall, and D. R. Leadley, Appl. Phys. Lett. 101, 172108 (2012).

${ }^{32}$ A. Nainani, B. R. Bennett, J. B. Boos, M. G. Ancona, and K. C. Saraswat, J. Appl. Phys. 111, 103706 (2012).

${ }^{33}$ L. Xia, J. B. Boos, B. R. Benett, M. G. Ancona, and J. A. del Alamo, Appl. Phys. Lett. 98, 053505 (2011).

${ }^{34}$ R. Winkler, Spin-Orbit Coupling Effects in Two-Dimensional Electron and Hole Systems (Springer, New York, 2003). 